\title{
Biomechanical Stability of Transverse Connectors in the Setting of a Thoracic Pedicle Subtraction Osteotomy
}

\author{
Ronald A. Lehman, Jr., MD ${ }^{1,2}$; Daniel G. Kang, MD ${ }^{1,2}$; Scott C. Wagner, MD ${ }^{1,2}$; Haines Paik, \\ $\mathrm{MD}^{1}$; Mario J. Cardoso, $\mathrm{MD}^{3}$; Joshua D. Bernstock, MS, $\mathrm{MPH}^{2}$; Anton E. Dmitriev, $\mathrm{PhD}^{2}$ \\ The views expressed in this manuscript are those of the authors and do not reflect the official \\ policy of the Department of Army, Department of Defense, or U.S. Government. Four authors \\ are employees of the United States government. This work was prepared as part of their official \\ duties and as such, there is no copyright to be transferred.
}

\author{
Affiliations: \\ ${ }^{1}$ Department of Orthopaedics, Walter Reed National Military Medical Center, Bethesda, MD \\ 20889 \\ ${ }^{2}$ Division of Orthopaedics, Department of Surgery, Uniformed Services University of the Health \\ Sciences, Bethesda, MD 20889 \\ ${ }^{3}$ Department of Neurosurgery, Walter Reed National Military Medical Center, Bethesda, MD \\ 20889
}

\section{Corresponding and Reprint Author:}

Ronald A. Lehman, Jr., MD

Walter Reed National Military Medical Center

Department of Orthopaedic Surgery and Rehabilitation

8901 Wisconsin Avenue, Building 19, Room \#2101

Bethesda, MD 20889

Telephone: (301) 319-4818

Fax: (301) 319-2361

Email: armyspine@yahoo.com 


\section{STRUCTURED ABSTRACT}

Background Context: Transverse connectors (TC) are often used to improve the rigidity of posterior spinal instrumentation, as previous investigations have suggested that TC enhance torsional rigidity in long segment thoracic constructs. Posterior osteotomies, such as the pedicle subtraction osteotomy (PSO), are can used in severe thoracic deformities and provide a significant amount of correction; as a consequence, however, PSOs also induce three-column spinal instability. In theory, augmentation of longitudinal constructs with TC after a thoracic PSO may provide additional rigidity, but the concept has not been previously evaluated.

Purpose: To evaluate the biomechanical contribution of TC to the rigidity of a long-segment pedicle screw-rod construct after a thoracic PSO.

Study Design/Setting: In vitro fresh-frozen human cadaveric biomechanical analysis.

Methods: Seven human cadaveric thoracic spines were prepared and instrumented from T4-T10 with bilateral pedicle screws/rods and a PSO was performed at T7. Intact range of motion (ROM) testing was performed with non-destructive loading and analyzed by loading modality (axial rotation $[\mathrm{AR}]$, flexion/extension [FE], and lateral bending [LB]). ROM analysis was performed in the unaugmented construct, the construct augmented with one TC, and the construct augmented with two TC. No outside funding sources were utilized for this study. The authors have no potential conflicts of interest to report.

Results: Following PSO and an unaugmented longitudinal pedicle screw rod construct, T4-T10 (overall construct) and T6-T8 (PSO site) ROM was significantly reduced in all planes of motion compared to intact condition (AR: 11.8 vs 31.7 degrees; FE: 2.4 vs 12.3 degrees; 3.4 vs 17.9 degrees, respectively, $\mathrm{p}<0.05)$. Augmentation of longitudinal construct with either one or two TC did not significantly increase construct rigidity in flexion-extension or lateral bending 
1 compared to the unaugmented construct ( $\mathrm{p}>0.05$ ). In contrast, during axial rotation, global ROM

2 was significantly reduced by $43 \%$ and by $48 \%$ at T6-T8 (1.7 and 1.2 degrees vs 2.38 degrees,

3 respectively) following addition of one and two TC $(\mathrm{p}<0.05)$. One TC did not significantly

4 reduce torsional $\mathrm{ROM}$ from the intact state.

5 Conclusions: Two transverse connectors significantly improved torsional rigidity of the entire

6 construct and at the PSO site, with no differences in rigidity for flexion-extension and lateral

7 bending, or with the addition of only one transverse connector. In the setting of a PSO and long

8 segment pedicle screw-rod construct, augmentation with at least two transverse connectors

9 should be considered to improve torsional rigidity.

10 Key Words: Pedicle subtraction osteotomy; transverse connector; cross-link; biomechanical 11 stability; thoracic spine; torsional stability. 


\section{INTRODUCTION}

Transverse connectors (TC), also known as cross-links or transfixators, have frequently been utilized to enhance the fixation strength of modern spinal instrumentation. While biomechanical investigations have suggested a probable role for TC in enhancing torsional rigidity[1], the exact threshold of rigidity required to improve clinical outcomes and fusion rates remains controversial and unproven. While some authors have demonstrated a biomechanical advantage through the addition of TC to long-segment fusion constructs, their role in the management of severe sagittal/coronal imbalance following a spinal osteotomy remains largely unknown. The pedicle subtraction osteotomy (PSO) is a surgical technique indicated in patients with severe sagittal plane deformities; the PSO is one of several posterior-only spinal osteotomies that achieve correction in the sagittal plane by wedge resection of the posterior column. However, the osteotomy itself is inherently destabilizing to the spinal column, and requires rigid fixation posteriorly to minimize catastrophic neurologic failure[2, 3]. While not exceptionally common, there is a subset of spinal deformity patients with fixed sagittal imbalance that require such posterior osteotomies to optimize the surgical correction of their deformities[4-9]. Transverse connectors may play a pivotal role in patients requiring a PSO for the management of a fixed sagittal deformity. While PSO is a technique more commonly performed in the lumbar spine, recent literature has shown thoracic PSO can be performed safely with good improvements in regional thoracic sagittal alignment, while avoiding the potential morbidity of an anterior exposure[2, 3, 5-8, 10]. The thoracic PSO is unique, as the correction occurs through all three columns of the spine and includes resection of the costovertebral joints at the surrounding vertebral segments, and may therefore predispose it to a degree of instability not often encountered in other clinical scenarios[2]. The reported clinical outcomes after PSO are 
1 generally very good, with favorable correction of spinal alignment and improvement in patient-

2 related outcomes scores, but it is not uncommon for pseudarthroses to occur at different levels,

3 which may be related to the instability that occurs after the osteotomy $[5-8,11]$. While pedicle

4 screw constructs alone have been shown to provide adequate stability in the sagittal and coronal

5 planes, they are not as effective in limiting axial torsion; particularly in long-segment

6 instrumentation[12].

7 As discussed, the efficacy of TC is controversial and as such there have been conflicting

8 results reported within the literature. A number of biomechanical investigations have

9 demonstrated a progressive, incremental increase in torsional stiffness as well as lateral

10 bending[13-17]. Conversely, there are concerns regarding the efficacy of these devices, as

11 several other studies have failed to demonstrate any appreciable clinical benefits despite the

12 significant additional cost of each supplementary transverse connector[18]. Another

13 disadvantage includes instrumentation crowding, which has been proposed to result in higher

14 rates of pseudoarthrosis secondary to an overall decrease in the surface area available for

15 fusion[19, 20].

16 To our knowledge, no other studies have evaluated the impact of TC in the setting of a

17 PSO, in which three-column stability and costovertebral articulations have been violated. While

18 transverse connectors are frequently used as an adjunct to enhance overall fixation strength, their

19 application in this particular clinical setting is without precedent. As such, no clear guidelines

20 exist to aid in determining the number and/or location for placement of TC after a PSO.

21 Consequently, the primary objective of the current investigation was to evaluate the

22 biomechanical contribution of TC to the rigidity of a long-segment pedicle screw construct after 
1 a PSO in the thoracic spine. Furthermore, we evaluated the role of TC number and location in an

2 effort to determine their impact with regard to overall biomechanical stability.

4 MATERIALS AND METHODS

$5 \quad$ Specimen Preparation

Seven fresh-frozen cadaveric spines were harvested from human cadavers. The medical

7 history for each cadaver was reviewed to exclude specimens with any underlying primary or

8 secondary bone disease. The bone mineral density (BMD) of all specimens was determined with

9 dual-energy x-ray absorptiometry, and spines with osteoporotic BMD were excluded. Each

10 specimen was then carefully disarticulated at the T2-T3 level proximally and the T11-T12 level

11 distally, with care to preserve all native osseous anatomy. The spines were carefully dissected

12 free of surrounding soft tissue attachments, leaving all ligamentous structures intact.

13 Additionally, the anterior $75 \%$ of the rib cage was resected, while intercostal ligaments and rib

14 articulations were maintained. However, the entire rib cage could not be preserved, as doing so

15 would prevent the specimens from fitting inside the biomechanical testing apparatus. All

16 specimens were inspected both visually and radiographically to identify the presence of pre-

17 existing fractures and/or compromised osseous integrity. Specimens were stored at $-20^{\circ} \mathrm{C}$ until

18 further testing ensued. Prior to biomechanical experimentation, the specimens were allowed to

19 thaw to room temperature. Post thaw, specimens were secured at both the proximal and distal

20 ends to a fixation jig utilizing a polyester resin supplemented by transfixion wires. The

21 specimens were copiously moistened using $0.9 \%$ sodium chloride irrigation solution throughout

22 the procedure. 
Prior to instrumentation or osteotomy, all specimens were tested first in the intact state as

2 a control. All specimens were then instrumented with polyaxial pedicle screws bilaterally, from

3 T4-T6 and T8-T10 utilizing the $5.5 \mathrm{~mm}$ titanium Legacy Spinal Fixation System (Medtronic,

4 Memphis, TN) by an experienced, fellowship-trained spine surgeon. All screws were inserted

5 with the technique outlined by Kim et al[21]. A pedicle subtraction osteotomy was then

6 performed at $\mathrm{T} 7$ according to the technique described by Bridwell et al.[3], with modifications

7 for the thoracic spine as described by O'Shaughnessy et al[5] (Figure 1). An important

8 modification for thoracic PSO includes removal of the rib heads bilaterally prior to completion of

9 the actual osteotomy, both at the PSO site and adjacent vertebral segments. During the

10 osteotomy, care was taken to avoid breach of the proximal and distal pedicles within proximity

11 of the osteotomy site. All specimens were visually and fluoroscopically evaluated to ensure the

12 absence of iatrogenic fracture propagation throughout the osteotomy site. Once this was

13 complete, $5.5 \mathrm{~mm}$ longitudinal rods were then appropriately contoured and secured to the pedicle

14 screws. Additional biomechanical testing was then performed in the following configurations:

1) No transverse connectors

2) One transverse connector placed directly below the level of the PSO (T8-T9)

Biomechanical Testing

Biomechanical evaluation was carried out utilizing the MTS 858 MiniBionix II system

20 configured with a custom six-degree-of-freedom Spine Simulator (MTS Systems, Inc.

21 Minneapolis, MN) (Figure 2), thereby allowing pure, unconstrained multidirectional load

22 application. Motion analysis was carried out with the use of specialized markers comprised of

23 infrared light emitting diodes (LED), which were individually and rigidly fixed to the anterior 
1 aspect of all vertebral levels from T4-T10. LED position in space was tracked using an optoelectronic motion analysis system (OptoTrak Certus, Northern Digital Inc., Waterloo, Ontario, Canada). Specimens were then exposed to non-destructive testing in all three planes of spinal motion: axial rotation (y-axis, $\pm 6 \mathrm{Nm}$ ), flexion/extension (x-axis, $\pm 6 \mathrm{Nm}$ ), and lateral bending (z-axis, $\pm 6 \mathrm{Nm}$ ). Spinal bending moments were applied for two loading and unloading cycles in each of the aforementioned planes.

\section{Data and Statistical Analysis}

Data obtained from the second load/unload cycle was utilized for final computational analysis. The peak ROM for each simulated loading moment was calculated as the sum of motion observed in the neutral (NZ) and elastic (EZ) zones. Full ROM (ROM = NZ + EZ) was reported for the whole construct (T4-T10) as well across the level of the osteotomy (T6-T8).

All data were shown as mean \pm 1 standard deviation. Statistical analyses included a repeated measure of Analysis of Variance (ANOVA) and test of simple effects incorporating Sidak's correction for multiple comparisons. Statistical results with a two-tailed $\mathrm{p}<0.05$ were defined as significant. The above mentioned analysis was conducted using SPSS software (SPSS, Version 13, SPSS Inc, Chicago, IL).

\section{RESULTS}

Axial Rotation ( \pm y axis)

Bilateral pedicle screw-rod fixation alone generated a $63 \%$ reduction in axial ROM throughout the entire construct (T4-T10) when compared to the intact spine $(11.8 \pm 4.6 \mathrm{vs}$ 31.6 \pm 11.1 degrees axial rotation, respectively, $\mathrm{p}<0.05$ ) (Table 1). While the addition of one TC immediately below the level of the osteotomy did trend toward an increase in the overall 
construct rigidity compared to the unaugmented construct, the difference was not statistically significant ( $8.8 \pm 2.6$ vs $11.8 \pm 4.6$ degrees axial rotation, respectively, $p>0.05)$. However, when compared to the unaugmented pedicle screw-rod construct, two TCs generated an additional $43 \%$ reduction in $\mathrm{ROM}(6.7 \pm 1.8$ vs $11.8 \pm 4.6$ degrees axial rotation, respectively, $\mathrm{p}<0.05)$ (Figure 3 ). Segmental range of motion was also evaluated at the level of the PSO (T6-T8). Similar to the findings observed throughout the entire construct, one TC alone did not significantly enhance rigidity compared to pedicle screw-rod fixation alone. However, with two TCs the construct had significantly decreased ROM (48\%) compared to the unaugmented construct $(\mathrm{p}<0.05)$ (Table 1) (Figure 4).

\section{Flexion/Extension ( $\pm x$ axis)}

Evaluation of flexion-extension loading demonstrated a significant $81 \%(\mathrm{p}<0.05)$ reduction in T4-T10 ROM (2.39 \pm 0.62 degrees $)$ with the unaugmented pedicle screw-rod construct compared to the intact spine (12.29 \pm 2.12 degrees) (Table 1) (Figure 3). The addition of one or two transverse connectors did not generate a significant improvement in overall construct rigidity $(\mathrm{p}<0.05)$. Similarly, there was a significant $83 \%(\mathrm{p}<0.05)$ reduction in segmental T6-T8 ROM at the level of the PSO with an unaugmented pedicle screw-rod construct compared to the intact spine, with no significant difference in flexion-extension ROM with the addition one or two transverse connectors $(\mathrm{p}>0.05)$ compared to the unaugmented longitudinal construct. (Table 1) (Figure 4)

\section{Lateral Bending ( $\pm z$ axis)}

Analysis of lateral bending ROM demonstrated results similar to flexion/extension testing. The unaugmented longitudinal construct significantly reduced $81 \%$ of intact spinal range of motion, with T4-T10 ROM decreased to 3.44 \pm 1.84 degrees $(\mathrm{p}<0.05)$. (Table 1) The addition 
1 of one or two TCs did not significantly increase the overall construct rigidity $(\mathrm{p}>0.05)$ compared

2 to the unaugmented longitudinal construct (Table 1) (Figure 3). Similarly, segmental ROM

3 (T6-T8) at the PSO site, had a significant decrease in ROM (84\%) with the additional of pedicle-

4 screw/rods alone compared to the intact spine, with no significant differences after the addition

5 of one or two TC compared to the unaugmented construct ( $p>0.05$ ) (Table 1) (Figure 4)

\section{DISCUSSION}

Our study demonstrated an unaugmented thoracic pedicle screw-rod construct in the setting of a PSO significantly reduced ROM in all planes of the entire construct and at the PSO site compared to the intact spine. In healthy individuals, previous biomechanical evaluation has suggested that axial torsion constitutes the primary plane of motion in the middle thoracic

12 spine[22], and this finding was corroborated by our data: intact axial rotation across T4-T10

13 averaged $31.7 \pm 11.1$ degrees, which was greater than both the flexion/extension $(12.3 \pm 2.1)$ and

14 lateral bending (17.9 \pm 4.8$)$ intact range of motion. Following supplemental fixation with

15 transverse connectors, the only significant findings compared to an unaugmented longitudinal

16 pedicle screw-rod construct was a $43 \%$ and $48 \%$ improvement in axial rotation rigidity of the

17 entire construct and at the PSO site, respectively, with the use of two TCs. The addition of one

18 TC trended toward improved axial rotation rigidity, but was not statistically significant. The

19 addition of either one or two TC did not provide additional biomechanical rigidity in

20 flexion/extension or lateral bending, as the general three-column stabilization achieved by

21 longitudinal pedicle-screw constructs was enough to reduce the global ROM in these two planes

22 of motion to approximately 3 degrees. Clinically, these results are relevant, in that the rate of

23 pseudarthrosis after PSO is relatively high, and while the ideal construct rigidity to promote 
1 fusion has never been experimentally established, it is reasonable to infer that improved rigidity

2 may contribute to the overall stability of the fusion, thereby reducing the rate of

3 pseudarthrosis[11].

$4 \quad$ There is no clear consensus regarding the contribution of transverse connectors to

5 biomechanical fixation strength. An initial study by Wood et al.[18] evaluated the role of TC in

6 long-segment fusions from T2-T11 and T2-L3. The authors compared multilevel fusion

7 constructs utilizing a combination of hooks, wires, and pedicle screws (with pedicle screws

8 inserted only at the distal ends of the construct). The authors found that the addition of one or

9 two TC offered no additional torsional rigidity to the construct, despite the fact that pedicle

10 screws were not instrumented at each vertebral level[18]. Utilizing a porcine model, Valdevit et

11 al.[23] found that transverse cross-links did not significantly alter torsional behavior.

Conversely, other studies have demonstrated more definite improvement with the use of

13 TC. Brodke et al.[13] evaluated the role of TC in short-segment fusion of a lumbar spine model.

14 The authors demonstrated that the biomechanical contributions of both pedicle screws and TC

15 increased incrementally as the length of the fusion construct increased[13]. Lynn et al.[16] also

16 found an increase in rigidity with the addition of one or two TC in a thoracolumbar model.

17 These findings were also obtained after an osteotomy was performed through all three columns

18 of their specimen; however, it should be noted that a true PSO was not performed, and a short-

19 segment fusion construct from T12-L2 was utilized. Another study by Lim et al.[17] showed

20 that TC improved both axial rotation and lateral bending stiffness. Kuklo et al.[14] evaluated the

21 contribution of TC in a long-segment fusion construct (T4-T10) with an all-pedicle screw

22 construct exclusively within the thoracic spine. Transverse connectors had no effect on construct

23 stiffness in flexion/extension or lateral bending[14], which are findings similar to those in our 
1 present study. With one TC, axial ROM was reduced by $21 \%$ compared to transpedicular

2 fixation alone, and two TC decreased axial ROM by an additional 14\%; furthermore, several test

3 configurations with TC at various sites generated no statistically significant changes in axial

4 ROM[14]. Based on their findings, the authors suggested that transverse connectors may be

5 most efficacious in the setting of extensive anterior or posterior column resection, such as for

6 severe deformity, infection, or tumor[14].

$7 \quad$ More recently, Majid et al.[24] showed that the biomechanical advantage of transverse

8 connectors is most significant in axial rotation, primarily when utilizing two TC at the proximal

9 and distal ends in a bilateral rod construct in the posterior cervical spine. Shaw et al[25] also

10 found that regardless of the degree of destabilization, TC effectively increased the rigidity of

11 rod-screw constructs in the cervical spine, with axial rotation again most significantly affected.

12 We also found that the placement of two transverse connectors reduced axial ROM, specifically

13 by an additional $43 \%$ when compared to transpedicular fixation alone, which falls in accordance

14 with these previous studies.

However, it is important to note that these prior studies have been limited to the cervical

16 spine, and their findings may not be entirely applicable to the thoracic spine. The thoracic region

17 is characterized by facets on both sides of the vertebral bodies for rib head articulation, and

18 facets on the transverse processes for articulation with the tubercles of the true ribs $\left(1^{\text {st }}\right.$ to $10^{\text {th }}$

19 ribs). The added stability provided by the rib cage likely alters the mechanics of the PSO, and

20 while we performed the PSO as originally described by Bridwell et al[3] with the thoracic

21 modifications proposed by O'Shaughnessy[5], differences still potentially exist between these

22 spinal regions. To our knowledge, there has been no correlation between the stabilizing effects of

23 TC in the cervical spine compared to the thoracic spine, nor have there been any studies 
1 evaluating whether a thoracic PSO destabilizes the thoracic spine to an extent similar to the

2 effects previously seen in the cervical spine[18-19]. Therefore, if these results are applicable to

3 the thoracic spine, it is likely only in the unique setting of a thoracic PSO with resection of the

4 adjacent level costovertebral articulations, which theoretically induces three-column instability

5 regardless of the surrounding thoracic cavity. However, this hypothesis has not been

6 experimentally shown and remains theoretical.

$7 \quad$ Our testing protocol involved resection of the sternum and the anterior $75 \%$ of the rib

8 cage due to mounting parameter restrictions for our testing apparatus, while maintaining

9 costovertebral articulations as well as surrounding intercostal ligaments and soft tissue. Doing so

10 introduces a potential limitation to our study conclusions, as numerous studies have shown the

11 biomechanical importance of the rib cage for spinal stability within the thoracic region (Table

12 1). It has been shown that the ribs account for up to $78 \%$ of thoracic spinal stability[26],

13 ultimately highlighting the rib cage as a "fourth column" of stability[27]. Therefore, our study

14 design may create an artificially unstable model, and the generalizability of our conclusions must

15 therefore be considered only in this context. Given this limitation, however, we believe that our

16 ability to evaluate changes in construct rigidity may be enhanced due to increased intact (non-

17 instrumented) specimen ROM in the setting of a partial rib cage. This is because, small

18 incremental differences in range of motion with the application of one or two TC may have been 19 more apparent.

Another limitation of our study design was the unique placement of the single TC at the

21 T8-T9 level. It may be argued that by changing the location of the single transverse connector,

22 one might induce an effect on the torsional rigidity of the construct. However, Kuklo et al.[14]

23 used a similar, albeit intact, thoracic spine model, and the authors make clear that changing the 
1 location of a single TC along a pedicle screw construct does not alter its torsional rigidity[14].

2 Additionally, we intentionally avoided placing the TC at the level of the PSO (T7), as it

3 theoretically would contribute to implant crowding and increased pseudoarthrosis in an actual

4 clinical scenario[19, 20]. This concern also informed our decision to limit the number of tested

5 TC to two; while axial rotation stiffness would be expected to improve with any additional TC,

6 the increased stability would likely result in diminished overall surface area available for

7 biologic fusion. Another weakness of the study protocol was the sequential analysis of the intact

8 specimen with incremental addition of one and then two TC. Although after each non-

9 destructive loading cycle the construct and instrumentation-bone interface were closely

10 evaluated, there may have potentially been changes in the viscoelastic properties of the specimen

11 or undetected instrumentation-bone interface loosening after multiple load-unload cycles, with

12 bias toward the later testing groups (two TC construct) to have decreased rigidity. Further, the

13 economic burden created by increasing the amount of instrumentation cannot be understated.

14 The increased cost associated with increased implant density has become an area of concern in

15 recent years, and many studies have evaluated the relationship between clinical outcomes and

16 overall cost of instrumentation [28]. Spinal implants are not inexpensive, and efforts to reduce

17 costs associated with spinal surgery are beneficial to society as a whole; however, if research

18 findings suggest that the addition of specific instrumentation may provide clinical benefits to

19 patients, it is reasonable to consider doing so on an individual patient basis. Finally, no outside

20 funding sources were utilized for this study. One author (R.A.L.) has received institutional grants

21 from the Defense Advanced Research Projects Agency (DARPA), Defense Medical Research

22 and Development Program (DMRDP), Depuy and Centinel Spine. There are no potential

23 conflicts of interest to report. 
2 PSO. Our findings are of clinical relevance, as much of the available biomechanical or finite

3 element literature evaluating transverse connectors focuses on fracture or instability models[15,

$429,30]$. However, burst fracture and corpectomy simulations do not result in three-column

5 disruption, and we hypothesize that the forces imparted onto the aforementioned constructs are

$6 \quad$ likely less than those seen following PSO. While pseudoarthrosis can certainly occur after any

7 spinal fusion procedure, the rates reported in limited series evaluating the results of PSO have

8 been as high as 29\%[2]. Additionally, the lack of published data addressing these concerns has

9 left surgeons without objective criteria to guide surgical decision-making. Determining the

10 location and number of TC required after correction of severe deformities has been anecdotal and 11 arbitrary at best.

12 In conclusion, our findings demonstrate that the application of two transverse connectors

13 to a long-segment fusion construct after PSO in the thoracic spine increases the torsional rigidity

14 of the entire construct and at the PSO site by $43 \%$ and $48 \%$, respectively. In the thoracic spine,

15 flexion/extension and lateral bending are not affected by transverse connectors. In a clinical

16 scenario in which a thoracic PSO is necessary for deformity correction, these biomechanical

17 considerations suggest incorporating two transverse connectors to enhance torsional rigidity of 18 pedicle screw-rod constructs. 
1

2 1. Dick JC, Zdeblick TA, Bartel BD, Kunz DN. Mechanical evaluation of cross-link designs

3 in rigid pedicle screw systems. Spine. 1997;22(4):370-5.

42 Bridwell KH, Lewis SJ, Lenke LG, Baldus C, Blanke K. Pedicle subtraction osteotomy

5 for the treatment of fixed sagittal imbalance. The Journal of bone and joint surgery American

6 volume. 2003;85-A(3):454-63.

7 3. Bridwell KH, Lewis SJ, Rinella A, Lenke LG, Baldus C, Blanke K. Pedicle subtraction

8 osteotomy for the treatment of fixed sagittal imbalance. Surgical technique. The Journal of bone

9 and joint surgery American volume. 2004;86-A Suppl 1:44-50.

10 4. Bridwell K. Decision Making Regarding Smith-Petersen vs. Pedicle Subtraction

11 Osteotomy vs. Vertebral Column Resection for Spinal Deformity. Spine. 2006;31(19

12 Suppl):S171-8.

13 5. O'Shaughnessy B A, Kuklo TR, Hsieh PC, Yang BP, Koski TR, Ondra SL. Thoracic

14 pedicle subtraction osteotomy for fixed sagittal spinal deformity. Spine. 2009;34(26):2893-9.

15 6. Yang BP, Ondra SL, Chen LA, Jung HS, Koski TR, Salehi SA. Clinical and radiographic

16 outcomes of thoracic and lumbar pedicle subtraction osteotomy for fixed sagittal imbalance.

17 Journal of neurosurgery Spine. 2006;5(1):9-17.

18 7. Bakaloudis G, Lolli F, Di Silvestre M, et al. Thoracic pedicle subtraction osteotomy in 19 the treatment of severe pediatric deformities. European spine journal : official publication of the 20 European Spine Society, the European Spinal Deformity Society, and the European Section of 21 the Cervical Spine Research Society. 2011;20 Suppl 1:S95-104.

22 8. Faundez A, Byrne F, Sylvestre C, Lafage V, Cogniet A, Le Huec JC. Pedicle subtraction 23 osteotomy in the thoracic spine and thoracolumbar junction: a retrospective series of 28 cases. 
1 European spine journal : official publication of the European Spine Society, the European Spinal

2 Deformity Society, and the European Section of the Cervical Spine Research Society. 2014.

39 Kelley BJ, Johnson MH, Vortmeyer AO, Smith BG, Abbed KM. Two-level thoracic

4 pedicle subtraction osteotomy for progressive post-laminectomy kyphotic deformity following

5 resection of an unusual thoracolumbar intradural extramedullary tumor. Journal of neurosurgery

$6 \quad$ Pediatrics. 2012;10(4):334-9.

7 10. Pellise F, Vila-Casademunt A. Posterior thoracic osteotomies. European journal of

8 orthopaedic surgery \& traumatology : orthopedie traumatologie. 2014.

9 11. Bridwell KH, Lewis SJ, Edwards C, et al. Complications and outcomes of pedicle

10 subtraction osteotomies for fixed sagittal imbalance. Spine. 2003;28(18):2093-101.

11 12. Lim TH, An HS, Hong JH, et al. Biomechanical evaluation of anterior and posterior

12 fixations in an unstable calf spine model. Spine. 1997;22(3):261-6.

13 13. Brodke DS, Bachus KN, Mohr RA, Nguyen BK. Segmental pedicle screw fixation or 14 cross-links in multilevel lumbar constructs. a biomechanical analysis. The spine journal : official 15 journal of the North American Spine Society. 2001;1(5):373-9.

16 14. Kuklo TR, Dmitriev AE, Cardoso MJ, Lehman RA, Jr., Erickson M, Gill NW.

17 Biomechanical contribution of transverse connectors to segmental stability following long 18 segment instrumentation with thoracic pedicle screws. Spine. 2008;33(15):E482-7.

19 15. Wahba GM, Bhatia N, Bui CN, Lee KH, Lee TQ. Biomechanical evaluation of short20 segment posterior instrumentation with and without crosslinks in a human cadaveric unstable 21 thoracolumbar burst fracture model. Spine. 2010;35(3):278-85. 
1 16. Lynn G, Mukherjee DP, Kruse RN, Sadasivan KK, Albright JA. Mechanical stability of

2 thoracolumbar pedicle screw fixation. The effect of crosslinks. Spine. 1997;22(14):1568-72;

3 discussion 73.

4 17. Lim TH, Kim JG, Fujiwara A, et al. Biomechanical evaluation of diagonal fixation in 5 pedicle screw instrumentation. Spine. 2001;26(22):2498-503.

6 18. Wood KB, Wentorf FA, Ogilvie JW, Kim KT. Torsional rigidity of scoliosis constructs.

$7 \quad$ Spine. 2000;25(15):1893-8.

8 19. Esses SI. Mechanical stability of thoracolumbar pedicle screw fixation: the effects of 9 crosslinks. Spine. 1997;(22):1573.

10 20. Kim YJ, Bridwell, K.H., Lenke, L.G., Rinella, A.S., Edwards, C. 2nd. Pseudoarthrosis in 11 primary fusions for adult idiopathic scoliosis: incidence, risk factors, and outcome analysis.

12 Spine. 2005;30(4):468-74.

13 21. Kim YJ, Lenke LG, Bridwell KH, Cho YS, Riew KD. Free hand pedicle screw placement 14 in the thoracic spine: is it safe? . Spine. 2004;29(3):333-42.

15 22. White AA, Panjabi, M.M. Clinical Biomechanics of the Spine, 2nd Ed.: Lippincott 16 Williams and Wilkins; 1990.

17 23. Valdevit A, Kambic HE, McLain RF. Torsional stability of cross-link configurations: a 18 biomechanical analysis. The spine journal : official journal of the North American Spine Society. $19 \quad 2005 ; 5(4): 441-5$.

20 24. Majid K, Gudipally M, Hussain M, Moldavsky M, Khalil S. The biomechanical effect of 21 transverse connectors use in a pre- and postlaminectomy model of the posterior cervical spine: an 22 in vitro cadaveric study. Spine. 2011;36(26):E1694-701. 
1 25. Shaw MN, Morel EP, Utter PA, et al. Transverse connectors providing increased stability

2 to the cervical spine rod-screw construct: an in vitro human cadaveric study. Journal of

3 neurosurgery Spine. 2011;14(6):719-25.

$4 \quad$ 26. Brasiliense LB, Lazaro BC, Reyes PM, Dogan S, Theodore N, Crawford NR.

5 Biomechanical contribution of the rib cage to thoracic stability. Spine. 2011;36(26):E1686-93.

6 27. Berg EE. The sternal-rib complex. A possible fourth column in thoracic spine fractures.

$7 \quad$ Spine. 1993;18(13):1916-9.

8 28. Yang S, Jones-Quaidoo SM, Eager M, et al. Right adolescent idiopathic thoracic curve

9 (Lenke $1 \mathrm{~A}$ and $\mathrm{B}$ ): does cost of instrumentation and implant density improve radiographic and

10 cosmetic parameters? European spine journal : official publication of the European Spine

11 Society, the European Spinal Deformity Society, and the European Section of the Cervical Spine

12 Research Society. 2011;20(7):1039-47.

13 29. Alizadeh M, Kadir MR, Fadhli MM, et al. The use of X-shaped cross-link in posterior 14 spinal constructs improves stability in thoracolumbar burst fracture: a finite element analysis.

15 Journal of orthopaedic research : official publication of the Orthopaedic Research Society. $16 \quad 2013 ; 31(9): 1447-54$.

17 30. Lazaro BC, Deniz FE, Brasiliense LB, et al. Biomechanics of thoracic short versus long 18 fixation after 3-column injury. Journal of neurosurgery Spine. 2011;14(2):226-34. 


\section{FIGURE LEGEND}

2 Figure 1. Pedicle subtraction osteotomy performed at T7 before (left) and after (right) closing

3 the defect to achieve sagittal correction.

4 Figure 2. Anterior view of a thoracic specimen undergoing flexibility testing with a six-degree-

5 of-freedom spine simulator.

6 Figure 3. Range of motion recorded from T4-T10 during loading in three planes of motion

7 (axial, flexion/extension, lateral bending). Error bars indicate 1 SD. * - indicates statistically

8 significant differences from the intact and pedicle screw-only specimens $(\mathrm{p}<0.05)$.

9 Figure 4. Range of motion recorded at the osteotomy level (T6-T8) during loading in three

10 planes of motion (axial, flexion/extension, lateral bending). Error bars indicate $1 \mathrm{SD}$. * -

11 indicates statistically significant differences from the intact and pedicle screw-only specimens (p

$12<0.05)$. 
Table 1. Multidirectional Flexibility Testing: ROM Data (Degrees)

$\underline{\text { Axial Rotation }}$

T4-T10 T6-T8

\section{Intact}

Mean

31.65

9.10

$11.07 \quad 1.54$

SD

Connector

\section{No Transverse Connector}

Mean

$11.84^{\dagger}$

$2.38^{\dagger}$

$4.56 \quad 1.06$

Flexion/Extension

Lateral Bending

SD

\section{Connector}

\section{Transverse Connector}

Mean

8.78

1.71

$2.62 \quad 0.54$

SD

T4-T10 T6-T8

T4-T10 T6-T8

\section{Transverse Connectors}

$\begin{array}{lcccccc}\text { Mean } & 6.71^{\dagger \dagger} & 1.23^{\dagger \dagger} & 2.27 & 0.81 & 3.36 & 0.68 \\ \text { SD } & 1.81 & 0.56 & 1.23 & 0.38 & 1.12 & 0.38\end{array}$

$\uparrow$ Significant difference compared to Intact ROM $(\mathrm{p}<0.05)$

$\dagger \dagger$ Significant difference compared to No Transverse Connector $(\mathrm{p}<0.05)$ 


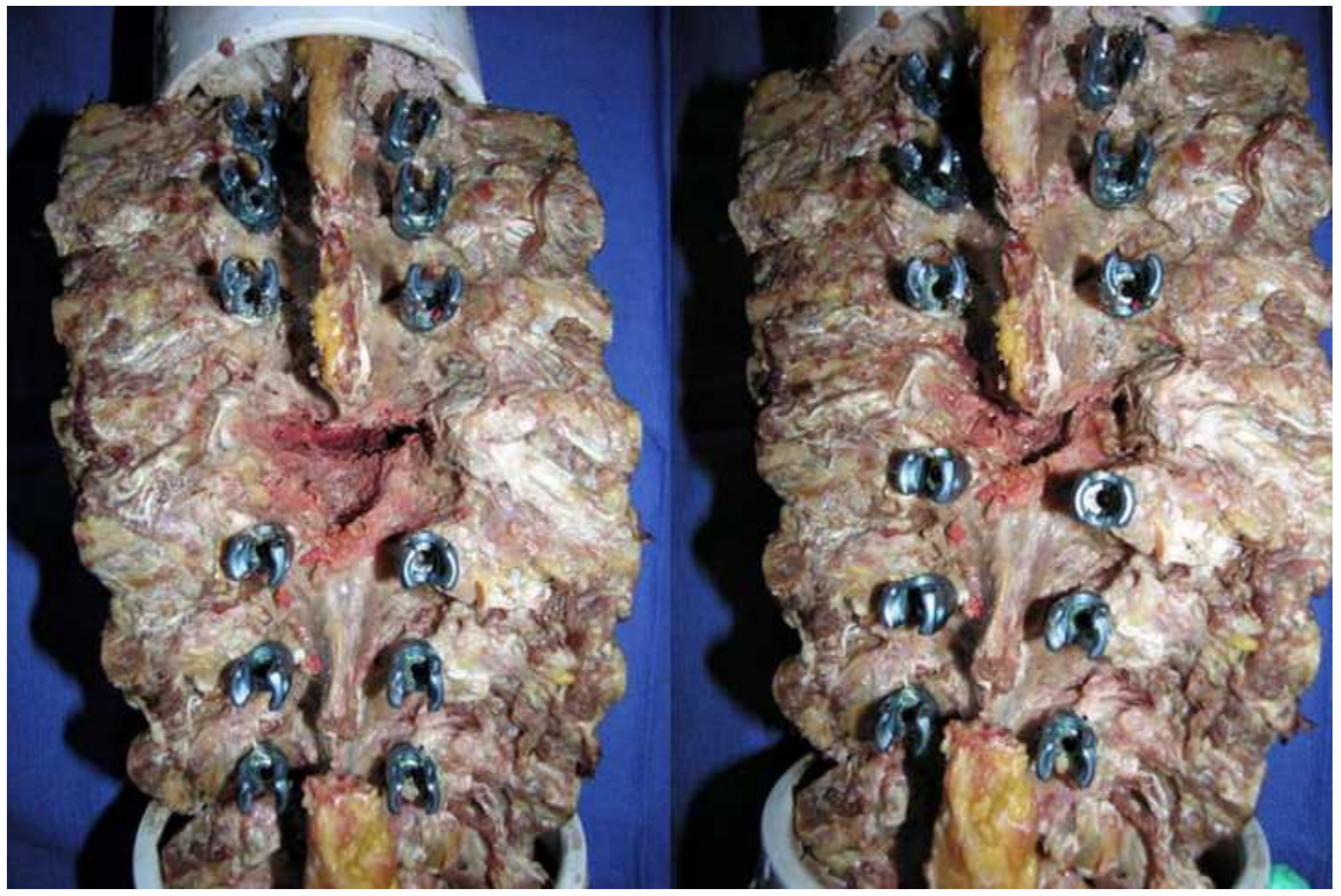

\section{ratset}

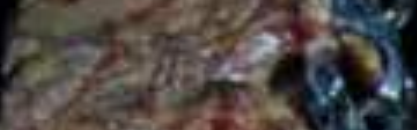

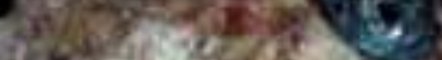

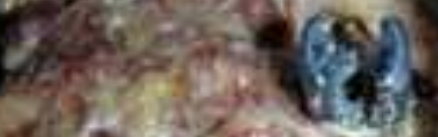

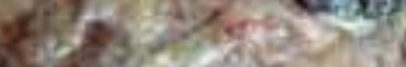
s.

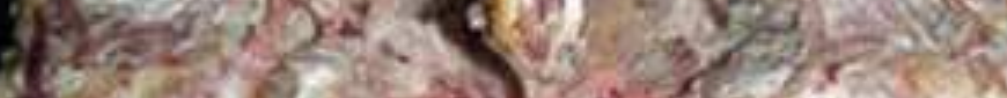

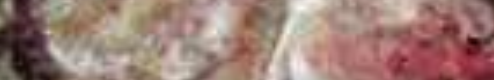

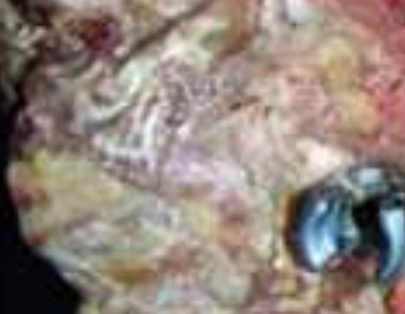
gevpons

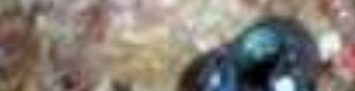
ary 829

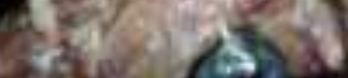
$\rightarrow 30$ is sor mat

(a)

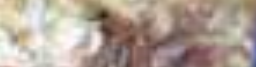
$x_{b}=2$ $\frac{1}{2} 8$ 政 (

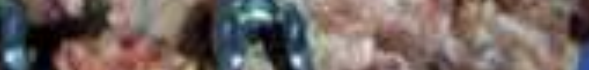
astatis:

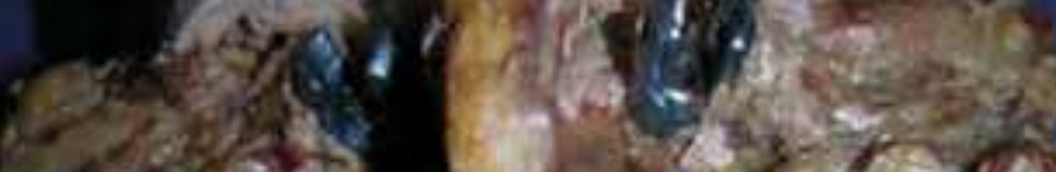

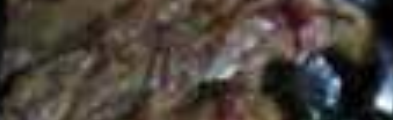

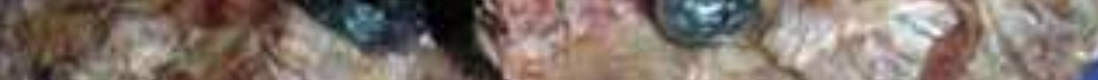
2.

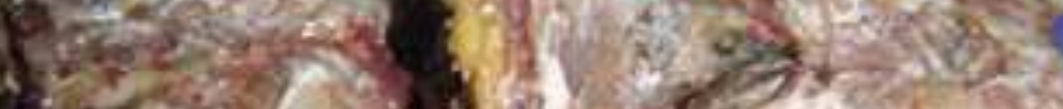

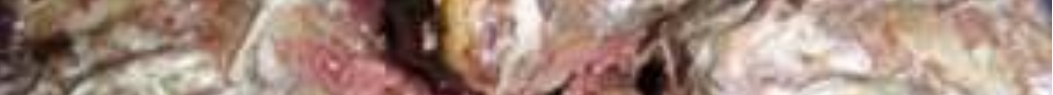
Nith

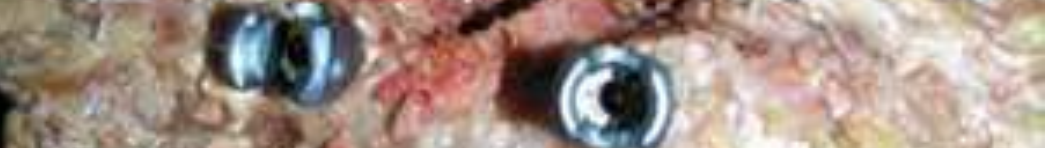

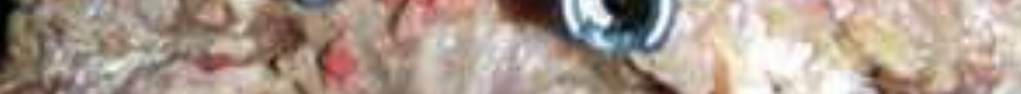

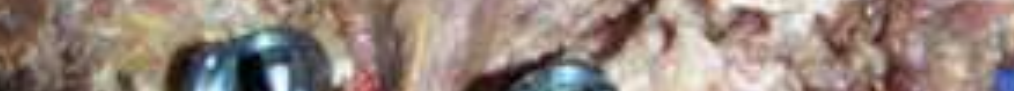

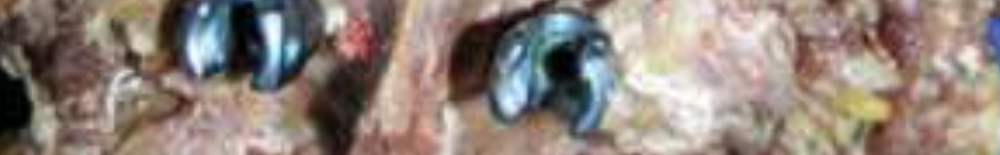

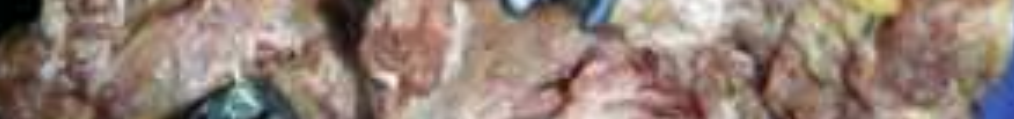

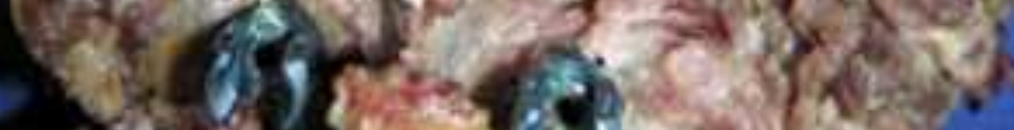
(6) $(10)$ 


\section{Multidirectional Flexibility Testing: T4-T10}

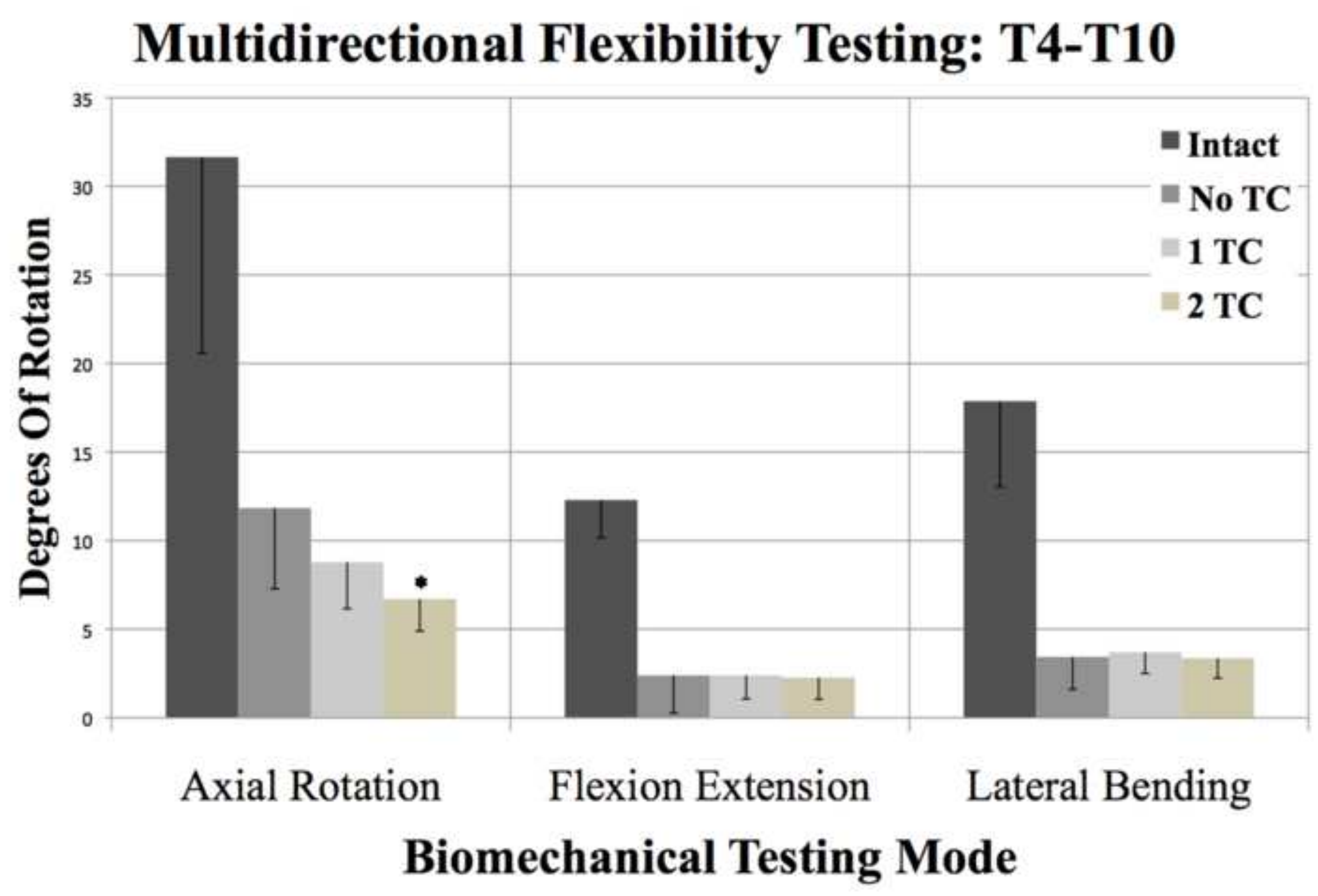

Axial Rotation Flexion Extension Lateral Bending Biomechanical Testing Mode 


\section{Multidirectional Flexibility Testing: T6-T8}

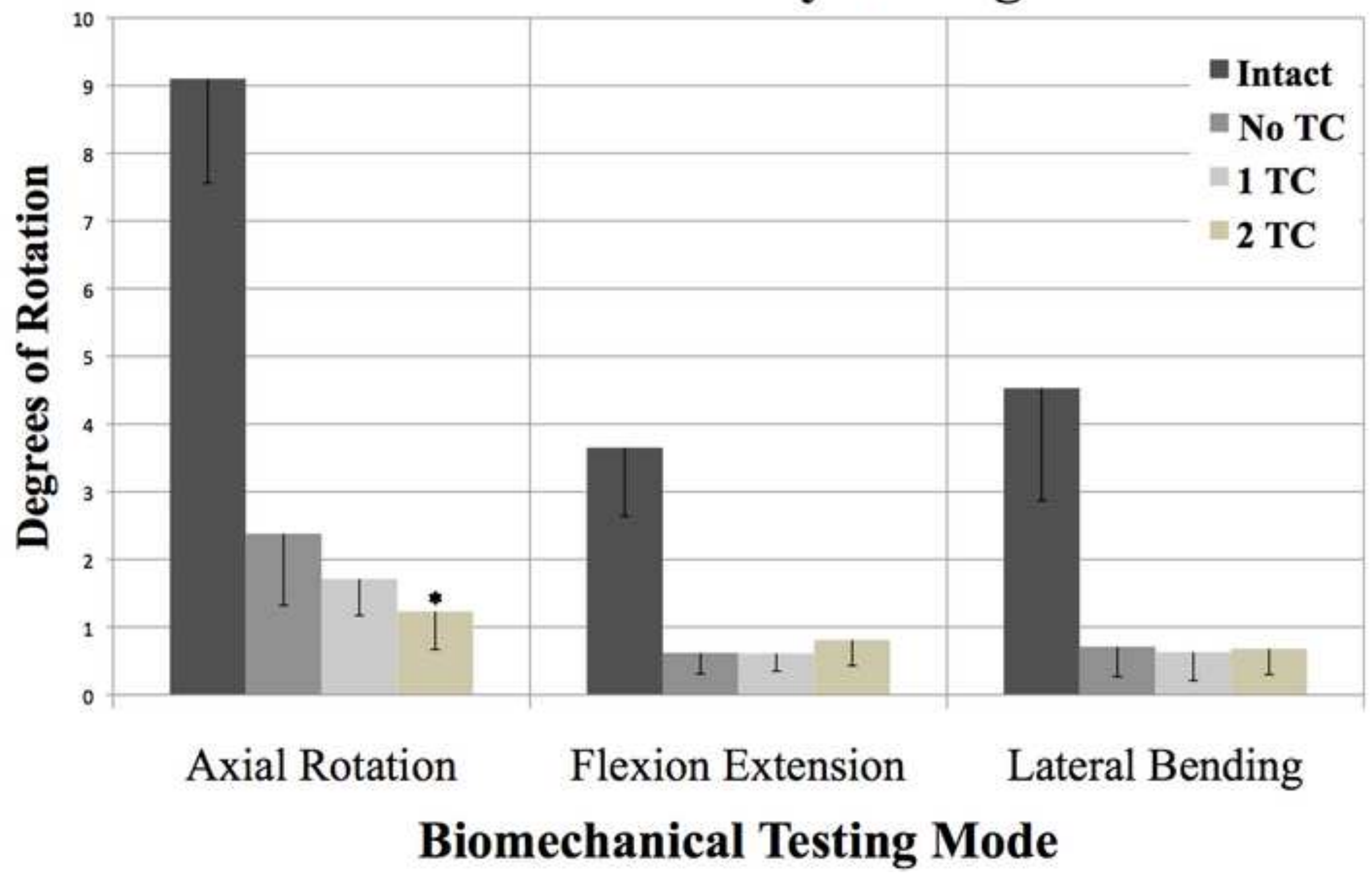

